\title{
Developing a Fall Detection Technology for Mobility and System Level
}

\author{
S. Divya \\ Assistant Professor, Department of Computer Science, \\ King College of Arts and Science, Namakkal, Tamil Nadu, India \\ E-Mail: divyasundarampillai@gmail.com
}

\begin{abstract}
Smartphone's are programmable and embed various sensors; these phones have the potential to change the way how healthcare is delivered. Fall detection is definitely one of the possibilities. Injuries due to falls are dangerous, especially for elderly people, diminishing the quality of life or even resulting in death. This study presents the implementation of a fall detection prototype for the Android-based platform. The proposed system has three components: sensing the accelerometer data from the mobile embedded sensors, learning the relationship between the fall behavior and the collected data, and alerting preconfigured contacts through message while detecting fall. We adopt different fall detection algorithms and conduct various experiments to evaluate performance. The results show that the proposed system can recognize the fall from human activities, such as sitting, walking and standing, with $72.22 \%$ sensitivity and $73.78 \%$ specificity. The experiment also investigates the impact of different locations where the phone attached. In addition, this study further analyzes the trade-off between sensitivity and specificity and discusses the additional powers consumption of the devices.
\end{abstract}

Keywords: Fall Detection, Sensitivity, Android SDK andIDE, Database, Customer Care

\section{INTRODUCTION}

Today's smart phones or mobile phones serve as the central computing and communication device in people's lives. Such a trend is inevitable in the real world due to the tremendous growth in recent years of new smart devices, such as iPhones, i-Pads, and Android-based platform, which is expected to continue. These phones embed various sensors, including accelerometer, digital compass, GPS, and camera, enabling new applications in various domains such as healthcare, social networks, and environmental monitoring. This study focuses on healthcare domain. One good example of these systems is fall detection. This is because falls are a major health risk for elderly people, diminishing the quality of life or even resulting in death. The fall detection system can be classified into four categories: image-based systems, database-based approach, context-aware techniques and acceleration-based detection. Each approach has its limitations and advantages. For example, the advantage of image-based and context-aware approaches is an accurate detection rate; the database-based approach stores various sensed user behavior into a database for various activities. The acceleration-based detection is the most widely used method, as current high-level devices build in the acceleration sensors.
The proposed system has three central components: sensing, learning, and alerting. In the first component, we take the advantages of the database-based approach to collect realistic fall data. That is, we collect the real accelerometer data from the mobile embedded sensors and record the corresponding user behavior to determine the required parameters. In the second component, the proposed system learns the relationship between the fall behavior and the collected data. In the third component, the mobile phones alert pre-configured emergency contacts through message. The experiment further investigates the impact of different locations where the phone attached, including chest, waist, and thigh. Finally, we conduct extensive experiments to evaluate the extra power consumption resulting from the fall detection software.

\section{SYSTEM ANALYSIS}

\section{A. Existing System}

The major problem with existing commercial products and academic research is that they have deficiencies that hinder pervasive fall detection. The base must be installed somewhere indoors and the portable sensor must be attached to a belt at the waist. Once the base receives the signal from the sensor indicating a fall, it can automatically communicate with a preset emergency contact using the fixed phone. However, the maximum distance between the sensor and the base is limited. Fall detection can only be conducted within a small indoor environment and elderly people may easily forget to bring the sensor with them, as it is an extra device that they seldom use in daily life. Furthermore, these products are expensive.

1. Drawbacks - Monitoring a person's movement with accuracy of intent is not easy. Fall detection technology is reported to trigger what scientists call "false positives" instances when the alarm is triggered but not by a fall.

For example:

a. Lying still while taking a nap may cause the monitor to think that a fall has occurred.

b. Slight tripping or a similar jerky movement may cause the monitor to detect a false fall.Vigorous exercise may confuse the monitoring device and cause the false alarm. 


\section{B. Proposed System}

This study proposes a low-cost fall detection system, using the existing devices and wireless technology, without the need for hardware modification, environmental setup, and wearing external sensors. The proposed system has three central components: sensing, learning, and alerting. In the first component, we take the advantages of the databasebased approach to collect realistic fall data. That is, we collect the real accelerometer data from the mobile embedded sensors and record the corresponding user behavior to determine the required parameters. In the second component, the proposed system learns the relationship between the fall behavior and the collected data. In this step, we utilize different fall features, including vertical and total acceleration, to design different fall detection algorithms [12]. We also measure the performance in both sensitivity and specificity while considering their trade- off [14]. In the third component, the mobile phones alert pre- configured emergency contacts through message. We further design an interface which allows users to manually disable the alert to avoid false positive and to reduce transmission costs.

\section{Advantages}

a. SMS alerts have been added, users can now send an SMS when fall or Inactivity occur.

b. Twitter \#tags are now supported for custom alerts.

c. A user preference has been added to allow the reporting of GPS locations in alerts.

d. Bug fix: GPS coordinates are now reported correctly.

e. Version 1.5 Algorithm refinements. Changed default calibration data.

f. Version 1.4 Added partial wake lock for compatibility purposes.

g. Version 1.3 Alert fix.

h. Version 1.2 Improvements on handling power off button. Fix regarding wake lock.

i. Version 1.1Changed default calibration settings.

j. Version 1.0 Interface changed to show the active emergency contacts.

k. Version 0.9 cancels false alarms more accurately.

l. Version 0.8 Fix regarding location services.

\section{SYSTEM DESIGN AND DEVELOPMENT}

Android is a software platform and operating system for mobile devices, based on the Linux kernel, and developed by Google and later the Open Handset Alliance. It allows developers to write managed code in the Java language, controlling the device via Google-developed Java libraries. Applications written in $\mathrm{C}$ and other languages can be compiled to ARM native code and run, but this development path is not officially supported by Google. The unveiling of the Android platform on 5 November 2007 was announced with the founding of the Open Handset Alliance, a consortium of 48 hardware, software and telecom companies devoted to advancing open standards for mobile devices. Google released most of the Android code under the Apache license, a free-software and open source license. Open Handset Alliance, is a consortium of several companies which include Google, HTC, Intel, Motorola, Qualcomm, T-Mobile, Sprint Nextel and NVIDIA, ... These companies which aim to develop technologies that will significantly lower the cost of developing and distributing mobile devices and services. The Android platform is the first step in this direction -- a fully integrated mobile "software stack" that consists of an operating system, middleware, user-friendly interface and applications.First and foremost, android is a software stack for mobile devices. This means that high on the list of priorities is the preservation of battery power and the efficient management of limited memory resources. There are five distinct layers to the Android system stack: Android is not a single piece of hardware; it's a complete, end-to-end software platform that can be adapted to work on any number of hardware configurations. Everything is there, from the boot loader all the way up to the applications.

\section{A. Operating System}

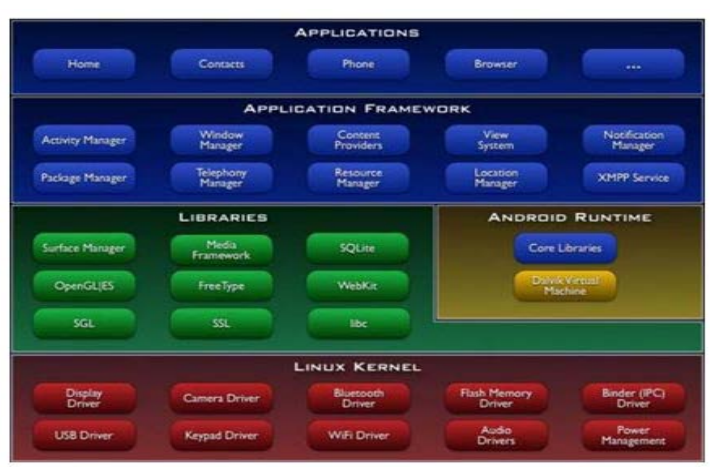

Fig.1 Architecture of Android OS

Android uses Linux for its device drivers, memory management, process management, and networking. However you will never be programming to this layer directly.

Network Connectivity:It supports wireless communications using:

1. GSM mobile-phone technology

2. $3 G$

3. Edge

4. $802.11 \mathrm{Wi}-F i$ networks

Development Requirements:Developing an application on the Android platform requires the following:

1. knowledge of programming in Java

2. knowledge of XML (optional but recommended)

3. Android SDK (requires x86 OS like Windows, Linux, Mac; JDK version >=5)

4. Eclipse IDE (at least version 3.3) with Android

5. Development Tools (ADT) plug-in (optional but recommended) 
6. Android powered smart-phone (optional for testingpurposes).

Notice that JavaME and JavaSE applications aren't runnable on Android as the class libraries as well as the generated byte code are different. The output design can be either displayed on a screen, VDU or a monitor. The design can be printed on paper as hard copy.Some of the prerequisites of output design are the output screens must be designed to be as attractive as possible and should also display the results in a simple and easy manner. The printed reports designed must also be as clear as possible and should be in a simple and easy-to-read way. The printed reports must also be designed to be as clear as possible and it should also make it easy for a user to find any information need. Results may be organized in tables. Result may also be displayed as graphs or chart. Complex ideas or concepts are often better displayed using a diagram. Some of the output design developing a Fall Detection Technology for Mobility and System Level to enter the fall detection is given in

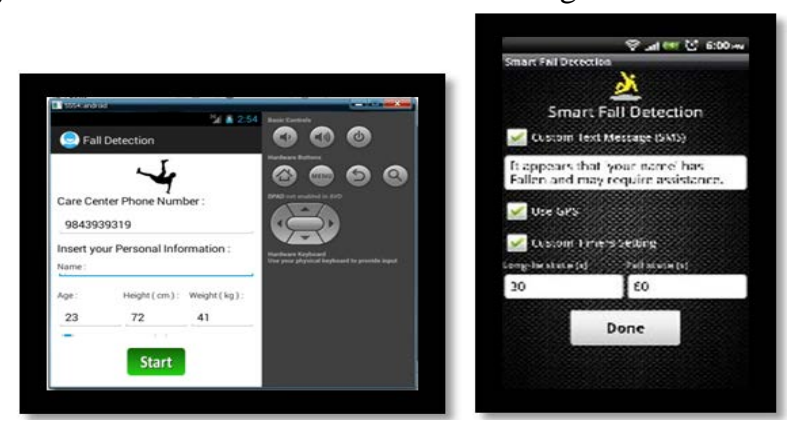

Fig.2 Output design for Fall Detection

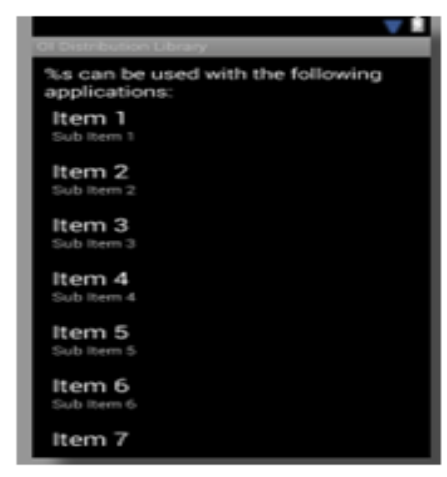

Fig.3 Database design for Fall Detection

\section{B. System DevelopmentModules}

1. Fall Down

2. SMS Send and Alarm

3. Event

\section{Module Description}

Fall Down:We implemented the fall detection system using Android- based devices, including one HTC Desire and two Tattoo mobile phones, with version 1.6 of the SDK platform. These phones were attached to different locations of human body, including the chest (pocket), waist (leather belt), and thigh (pocket). We used these phones to collect realistic three-axis acceleration and orientation measurements for different activities of daily living, including walking, sitting and standing up, and falling. Each data interval is 200 MS and we set a one-second time window to average these measurements. It shows a typical example of the total acceleration between different activities during 28 seconds. It demonstrates that a fall behavior is highly related to the acceleration data, resulting in a dramatically change and a peak acceleration value. There are four different users, with different height and ages, to perform the experiments. Each user continuously changed the activities for 28 seconds in a fixed area, and repeated for 30 times. The collected data was manually labeled as falling and other activities and divided into training and testing sets. The trainings data is used to find the threshold of the overall acceleration.

SMS Send and Alarm:In the emergency notification process, the mobile phone starts an alarm message on the screen and records the alarm time after detecting fall. When user receives the message, they can determine whether healthcare assistance is required or not. In this step, we further design an interface which allows users to manually disable the alarm to avoid false positive and to reduce transmission costs. If the user stops the alarm in time, then the algorithm backs to the monitoring stage. If not, the mobile phones alert pre-configured emergency contacts through message. We assume that the mobile phones contained the SIM card and can send message through the telecommunication company.

Event: The Event shows the sensitivity versus specificity in different phone-attached locations based on the overall acceleration. For each location, we adjust the thresholds and compute the sensitivity and specificity, as indicated by the markers of each line. This is also known as receiver operating characteristic curve. This clearly shows the tradeoff between sensitivity and specificity. A higher sensitivity is always gained at the expense of a low specificity. That means that determining the threshold should consider the balance between sensitivity and specificity. Moreover, this figure shows that the chest perform the best, achieving a $72.22 \%$ sensitivity and a $73.78 \%$ specificity at the same time. This is because when themobile phone is attached in a higher location, the change of acceleration is more significant to detect the fall.

\section{SYSTEM IMPLEMENTATION}

System implementation is the practice of creating or modifying a system to create a new business process or replace an existing business process. Technology integration is the practice of integrating multiple system activities to interact and generate information effectively. In both cases, the system development lifecycle (SDLC) is heavily integrated in this practices. A CPA professional may be involved in designing a system, performing end user testing, or training others how to utilize the financial system. Fall 
Detection data source is implemented through the java and SQLite system.

Three Types of implementation are

1. Implementation of new proposed system to replace the existing system.

2. Implementation of modified application to replace an existing system.

3. Implementation of derived system to replace the existing system.

The implementation phase involves mainly three steps, A. Planning:The implementation of the system involves people from different sections and it involves many problems. Co-operation from different developers was sought for successful implementation.

B. Training:Good training is to be provided to the users of the system. An operation manual provided with the system makes the process easier.

C. Changeover:It is the process of converting the existing system into new system. The new system was found easier to use and the developer were satisfied with reports generated by the new system. The proposed system can be further enhanced by providing the software service based on Android OS. If provided software management application will be efficiently. Not only software can be provided as a service mobile application our proposed system.

\section{CONCLUSION}

Falls are a major health risk for elderly people, diminishing the quality of life or even resulting in death. This study designs and implements a fall detection prototype for the Android-based platform. We propose a low-cost fall detection system, using the existing devices and wireless technology, without the need for hardware modification, environmental setup, and wearing external sensors. The proposed system has three central components: sensing the accelerometer data from mobile embedded sensors, learning the relationship between the fall behavior and collected data, and alerting pre- configured contacts through message while not receiving user's response. We adopt different algorithms to design fall detection algorithms, and conduct various experiments to evaluate performance. The results show that the proposed system can recognize the fall from human activities of daily living, such as sitting, walking and standing, with $72.22 \%$ sensitivity and $73.78 \%$ specificity. In addition, this study analyzes the trade- off between sensitivity and specificity by plotting receiver operating characteristic. The experiment further investigates the impact of different locations where the phone attached and also evaluates the extra power consumption on mobile phones.

\section{REFERENCES}

[1] N. Lane, E. Milosz, H. Lu, D. Peebles and A. Campbell”,A survey of mobile phone sensing”, Communications Magazine, IEEE, Vol. 48, No. 9, pp. 140-150, 2010.

[2] J. Dai, J. Tang, X. Bay, Z. Sheng, and D. Juan, "Mobile phone based drunk driving detection”,In International Conference on Pervasive Computing Technologies for Healthcare, pp. 1-8, 2010.

[3] S. Consul, D. W. McDonald, T. Tosco’s, M. Y. Chen, J. Froehlich. Harrison, P. Klansman, A. Lamarck, L. Ligand, R. Libby, I. Smith, and J. A. Landau, "Activity sensing in the wild: a field trial of befit garden”,In Proceeding of the twenty-sixth annual SIGCHI conference on Human factors in computing systems, CHI '08, pp. 1797-1806, 2008.

[4] D. Anderson, J. Keller, M. Subic, X. Chen, and Z. He, "Recognizing falls from silhouettes”,In Engineering in Medicine and Biology Society, 28th Annual International Conference of the IEEE, pp. 6388-6391, 2006.

[5] W.Y. Shih and J.C. Huang, "Speedup the multi-camera videosurveillance system for elder falling detection”,In International Conference on Embedded Software and Systems.

[6] [Online] Available: developer.android.com/.../android/fall detection/package-summary.html

[7] [Online] Available: www.android.com

[8] [Online] Available:www.developer.android.com/guide

[9] [Online] Available: www.openhandsetalliance.com

[10] [Online] Available: www.google.com/mobile/android

[11] [Online] Available: developer.android.com/guide/topics/wireless/ falldetection.html. 